\title{
Heavy flavour physics results from the ATLAS experiment
}

\author{
Zdeněk Doležal ${ }^{* \dagger}$ \\ Charles University in Prague, Faculty of Mathematics and Physics, \\ $V$ Holesovickach 2, Prague, The Czech Republic CZ180 00 \\ E-mail: dolezaleipnp.mff.cuni.cz
}

\begin{abstract}
ATLAS has a wide programme to study the production cross-section and decay properties of particles with beauty, as well as charmonium and bottomonium states. This paper covers the latest ATLAS results, including searches for excited $b$-hadrons, new decay modes of $b$-hadrons, indirect New Physics searches in rare $b$-hadron decays, as well as the study of mixing and CP violation in the $B_{s}$ system. We also review the results in the domain of charmonium production, including $J / \psi, \psi(2 S)$ and $\chi_{c}$ states, associated vector boson $+J / \psi$ production and a search for hidden-beauty state $X_{b}$, the bottomonium counterpart of $X(3872)$, in the $\Upsilon \pi \pi$ channel.
\end{abstract}

53rd International Winter Meeting on Nuclear Physics,

26-30 January 2015

Bormio, Italy

\footnotetext{
* Speaker.

${ }^{\dagger}$ On behalf of the ATLAS Collaboration
} 


\section{Introduction}

ATLAS is a general purpose detector built at the Large Hadron Collider at CERN, Geneva. Its main objective is to study the Higgs boson and search for physics beyond the Standard Model (BSM) in the high $p_{\mathrm{T}}$ domain. In addition to these topics it has a rich flavour physics program as well. Characteristics of flavour-related processes serve also as an important input to test QCD predictions for proton-proton $(p p)$ collisions. Measurements of properties of $b$-hadrons, CP Violation in $B$-decays and searches for exotic particles and rare decays have also been performed using data collected in $2011(\sqrt{s}=7 \mathrm{TeV})$ and $2012(\sqrt{s}=8 \mathrm{TeV})$ data taking periods (called Run 1).

\section{ATLAS Detector and Triggers}

A detailed description of the ATLAS detector can be found in [1]. Only the most important parts of the nominal detector relevant for flavour physics studies are described in this paper. The Inner Detector uses its magnetic field of $2 \mathrm{~T}$ to measure charged particle tracks in a pseudorapidity range of $|\eta|<2.5$. In the barrel region it consists of 3 pixel layers followed by 4 silicon strip detector layers and the Transition Radiation Tracker allowing to reach design momentum precision of $\sigma_{p_{\mathrm{T}}} / p_{\mathrm{T}}=0.05 \% p_{\mathrm{T}} \oplus 1 \%$. The Muon Spectrometer (MS) - the outermost detector of ATLAS - consists of four parts (monitored drift tubes, cathode strip chambers, thin-gap chambers and resistive plate chambers). It covers $|\eta|<2.7$ and due to its toroidal magnetic field it can measure transverse momenta with a nominal resolution of $\sigma_{p_{\mathrm{T}}} / p_{\mathrm{T}}=10 \%$ at $p_{\mathrm{T}}=1 \mathrm{TeV}$. This system also provides important muon triggers. Electromagnetic and hadronic calorimeters are placed between the tracker and the MS.

The ATLAS trigger consists of three levels, Level 1 (L1), Level 2 (L2) and the Event Filter (EF). L1 trigger is a hardware trigger based on the MS, Calorimeters and Minimum Bias Trigger Scintillators (MBTS). L2 and EF triggers are software triggers and are together called High Level Trigger (HLT).

\section{Observation of Excited $B_{c}^{ \pm}$Meson State}

The $B_{c}^{ \pm}$meson was first observed by the CDF experiment [2]. ATLAS has performed a search for excited states of the $B_{c}^{ \pm}$meson using $4.9 \mathrm{fb}^{-1}$ of $7 \mathrm{TeV}$ and $19.2 \mathrm{fb}^{-1}$ of $8 \mathrm{TeV} p p$ collision data [3]. A new state was observed, with mass consistent with the $B_{c}^{ \pm}(2 S)$ predictions. It is reconstructed in the decay to the $B_{c}^{ \pm}$meson and two oppositely charged pions. The $B_{c}^{ \pm}$was reconstructed through its decay to $J / \psi \pi^{+}, J / \psi \rightarrow \mu^{+} \mu^{-}$. The peaks were sought in the spectrum of the variable

$$
Q=m\left(B_{c}^{ \pm} \pi \pi\right)-m\left(B_{c}^{ \pm}\right)-2 m\left(\pi^{ \pm}\right)
$$

Here $m\left(B_{c}^{ \pm}\right)$is the reconstructed invariant mass of the $B_{c}^{ \pm}$candidate combined with two charged pion candidates. The peak in this distribution is clearly visible in Fig. 1. The newly observed state has mass of $(6842 \pm 4 \pm 5) \mathrm{MeV}$ with a $5.2 \sigma$ significance. 

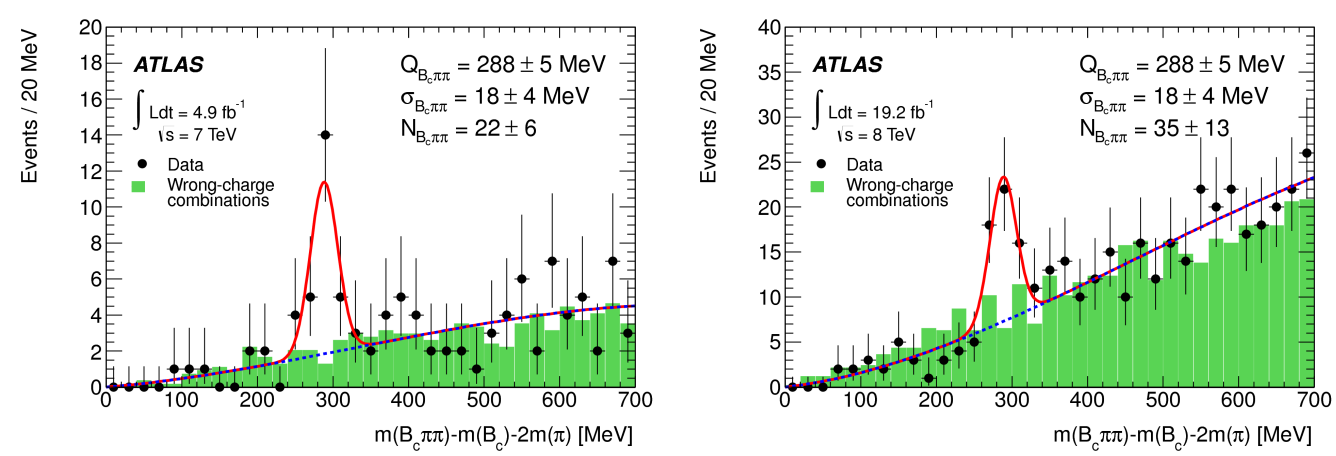

Figure 1: Distribution of $Q$ variable for the excited $B_{c}^{ \pm}$search (see text) for 2011 (left) and 2012 (right) data.

\section{New Physics Search in the $B_{s}^{0} \rightarrow J / \psi \phi$ Decay}

The decay channel $B_{s}^{0} \rightarrow J / \psi \phi$ is expected to be sensitive to BSM physics. The CP violation that is manifested here is due to an interference between direct decays and decays with $B_{s}^{0}-\bar{B}_{s}^{0}$ mixing. The CP violation can be quantified by the CP-violating phase $\phi_{s}$.

$$
\phi_{s}=-2 \arg \left[-\frac{V_{t s} V_{t b}^{*}}{V_{c s} V_{c b}^{*}}\right]
$$

It should be small according to the Standard Model, but can be much larger in many new physics models. This variable is usually plotted against the width difference $\Delta \Gamma_{s}=\Gamma_{L}-\Gamma_{H}$ of the light and heavy $B_{s}^{0}$ mass states. ATLAS has published a new measurement of $\phi_{s}$ with flavour tagging [5]. An unbinned maximum likelihood fit is performed on the selected events to extract the parameters of the decay. Fig. 2 shows $B_{s}$ mass and proper decay time projections of the PDF. The result of this ATLAS measurement is included in Fig. 3 together with results of other experiments as well as with the world average made by the Heavy Flavour Averaging Group (HFAG) [4]. One can see that all measurements as well as the average are consistent with the SM prediction.

\section{Search for the $X_{b}$ and Other Hidden Beauty States}

A number of anomalous particles have been observed in the charm and beauty spectra in past years, $X(3872)$ being the first one. The first observation by Belle [6] was quickly confirmed by other experiments [7]-[9]. The character of this hidden-charm state is still a subject of debate and various explanations have been proposed. Heavy-quark symmetry suggests the existence of an analogous state in the hidden beauty sector $-X_{b}$. ATLAS has performed a search for this state [10] in a corresponding decay channel

$$
X_{b} \rightarrow \pi^{+} \pi^{-} \Upsilon(1 S)\left(\rightarrow \mu^{+} \mu^{-}\right)
$$

The search was performed in a wide range around the predicted mass, i.e. $10-11 \mathrm{GeV}$, assuming a narrow state with 3-body decay characteristics. Known Upsilon states have been used as a reference and for validation, since they decay into the same final state

$$
\Upsilon(2 S, 3 S) \rightarrow \pi^{+} \pi^{-} \Upsilon(1 S)\left(\rightarrow \mu^{+} \mu^{-}\right)
$$



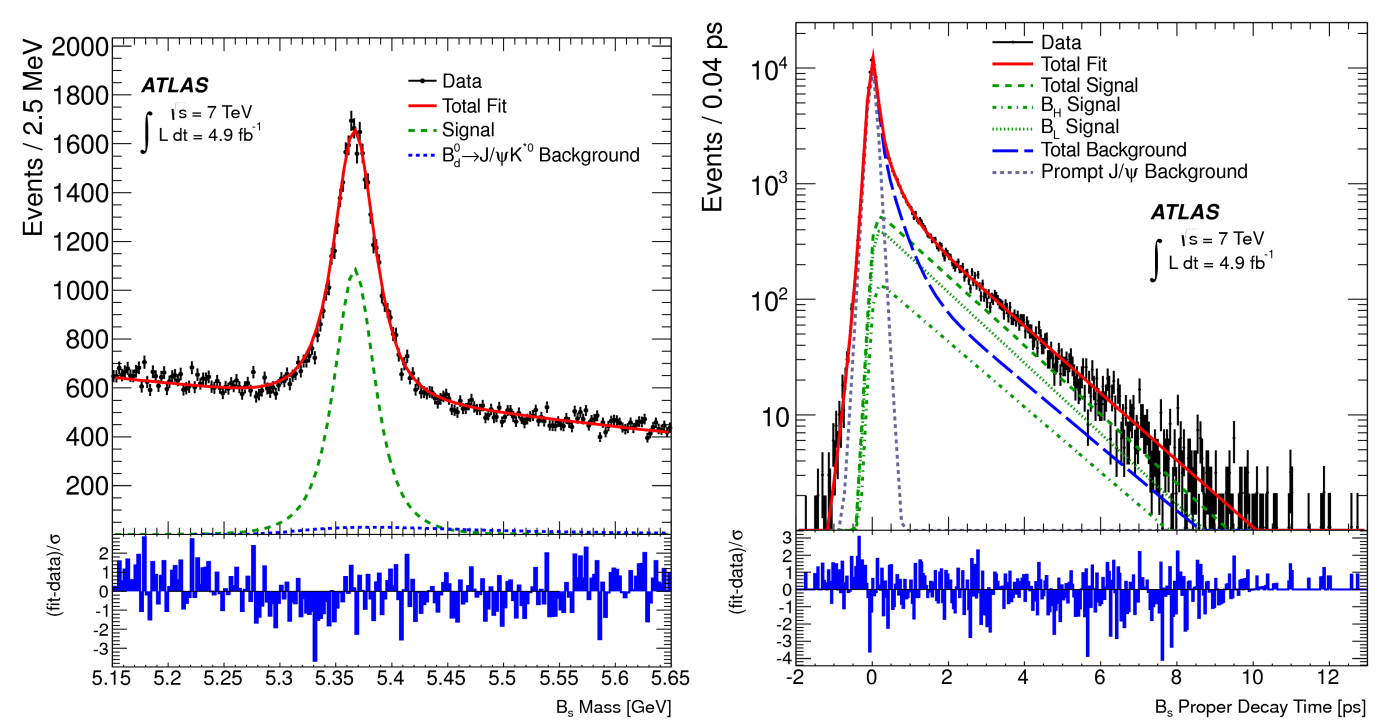

Figure 2: Mass fit (left) and proper decay time fit (right) projection for the $\mathrm{B}_{s}^{0} \rightarrow J / \psi \phi$ candidates.

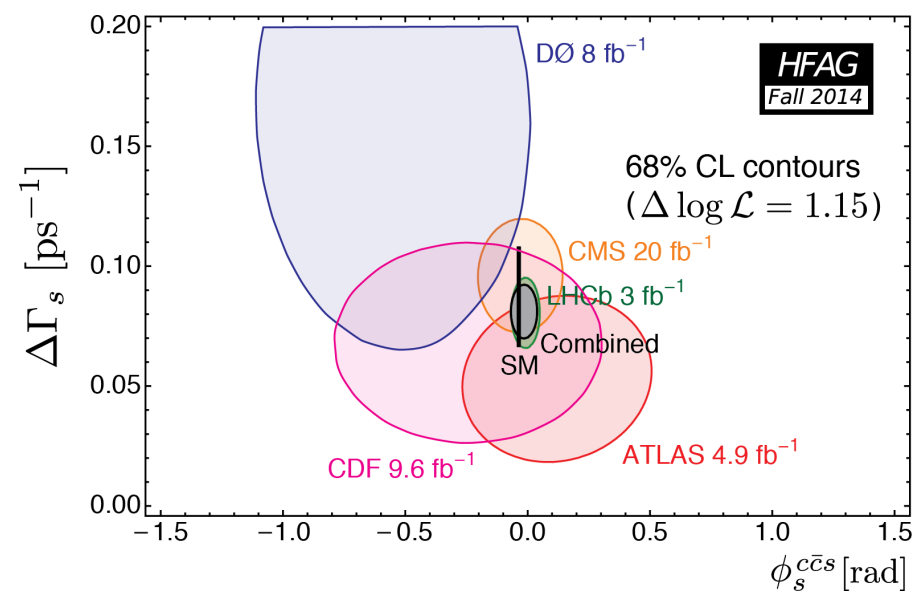

Figure 3: Overview of the measurements of the $B_{s}^{0} \rightarrow J / \psi \phi$ decay together with the Heavy Flavour Averaging Group (HFAG) [4] average of $\Delta \Gamma_{s}$ vs. $\phi_{s}$.

No signal has been found and upper limits on $R=(\sigma \mathcal{B}) /(\sigma \mathcal{B})_{2 S}$, the relative production rate of a hypothetical $X_{b}$ to that of $\Upsilon(2 S)$, were determined for each mass hypothesis (see Fig. 4). The limits are the most restrictive ones available above $10.1 \mathrm{GeV}$.

\section{Heavy Flavour Production}

\subsection{Production Cross-section of $\psi(2 S)$}

Despite many years of theoretical and experimental studies the production mechanisms of charmonia are far from being understood. This is due to a mixture of energy scales and the mass of the $c$-quark. New measurements of the $\psi(2 S)$ production cross-section can provide more insight into the processes involved and can constrain the existing models with precise data. The analysis presented here studies the prompt and non-prompt production of $\psi(2 S)$ decaying into 


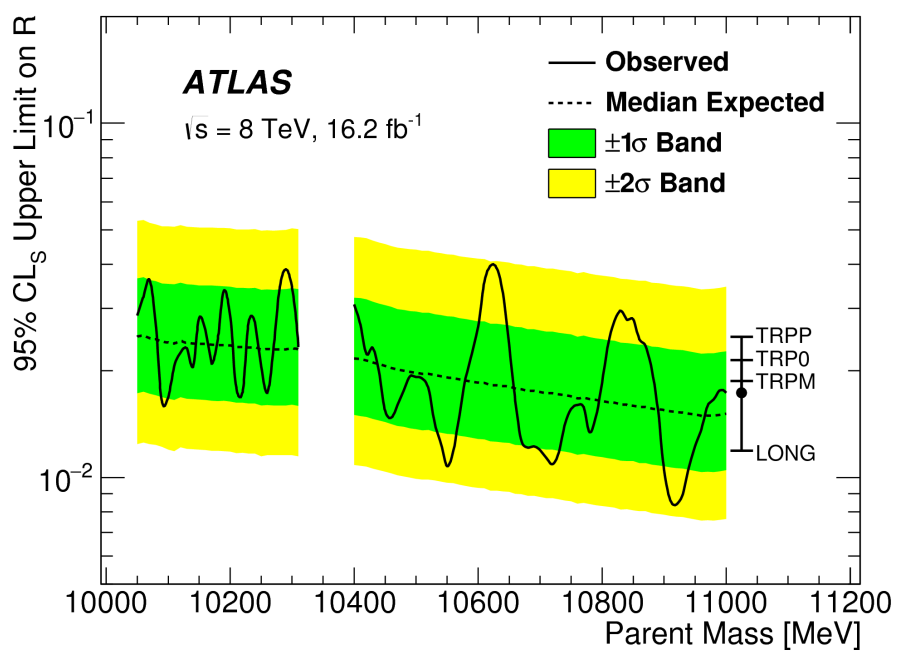

Figure 4: Observed $95 \% \mathrm{CL}_{s}$ upper limits (solid line) on the relative $X_{b}$ production rate $R$.
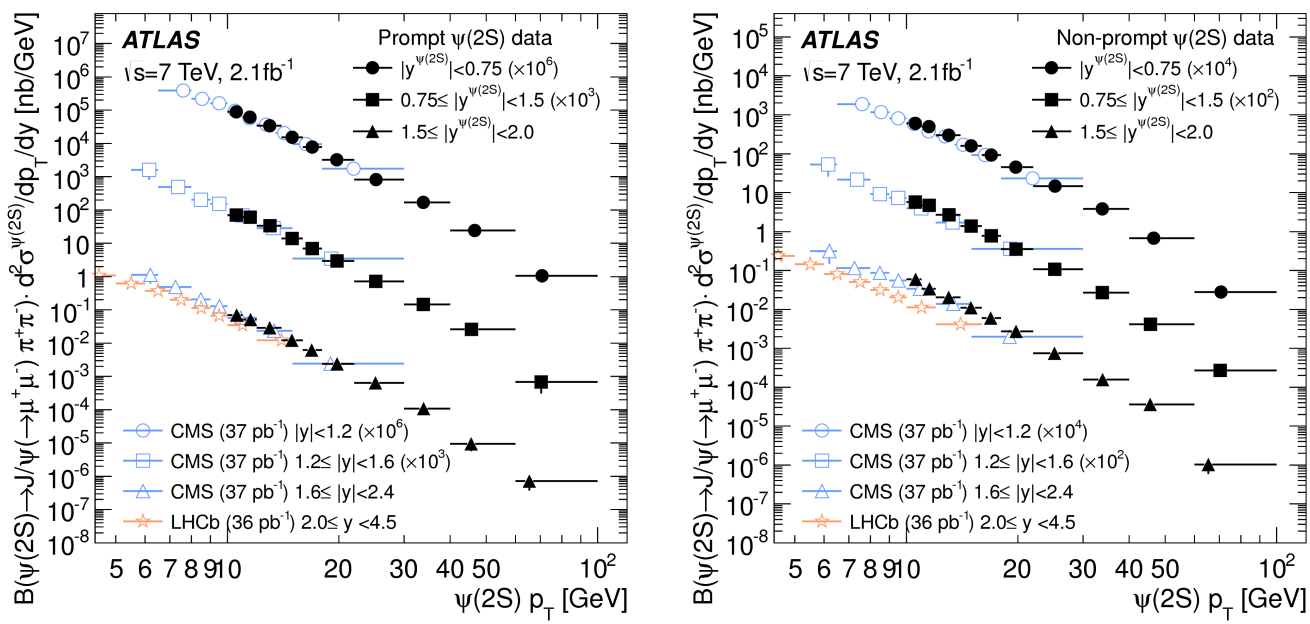

Figure 5: Prompt (left) and non-prompt (right) production vs. $\psi(2 S) p_{\mathrm{T}}$. CMS [13] and LHCb [14] results are shown for comparison

$J / \psi\left(\mu^{+} \mu^{-}\right) \pi^{+} \pi^{-}$. The prompt production comes directly from the QCD production mechanisms, while the non-prompt production goes via weakly decaying $b$-hadrons. The measurements yielded results in the $10-100 \mathrm{GeV} p_{\mathrm{T}}$ range and rapidities $|y|<2$. Cross-sections and theory-to-data ratios are shown in Figs. 5-6. One can see, that predicting prompt production results is still a difficult task for some models $\left(k_{T}, \mathrm{CS}\right.$ and CE), while NR QCD LO and NLO calculations agree with data very well except in the higher $p_{\mathrm{T}}$ region. In non-prompt production the available models do quite well.

A detailed comparison with a variety of theoretical models as well as with ALICE, CMS and LHCb measurements can be found at the original paper [12].

\subsection{Production of Heavy Quarkonia $\chi_{c 1}$ and $\chi_{c 2}$}

As explained in the previous section, quarkonia production studies are a very useful tool for 

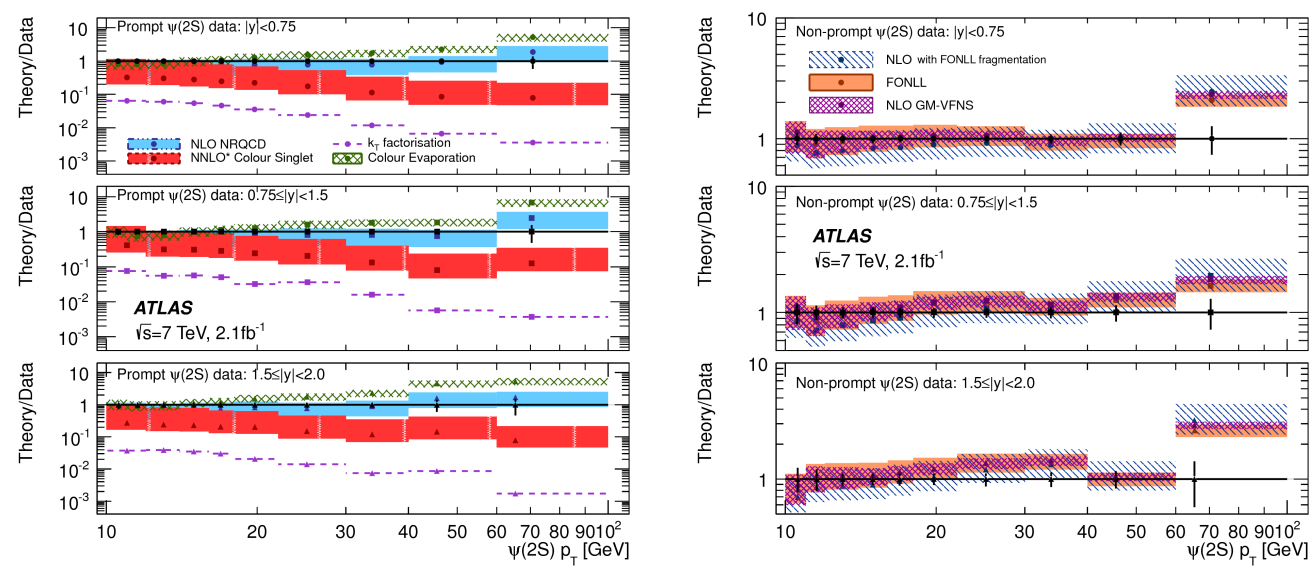

Figure 6: Ratios of the predicted to measured differential cross-sections for prompt (left) and non-prompt right) $\psi(2 S)$ production.

testing various QCD models. However the production of $J / \psi$ is accompanied by a large feed-down from $\chi_{c}$ and $\psi(2 S)$ decays. This feed-down production is currently estimated to be about $25 \%$. Hence understanding the production of the triplet $P$-wave states $\chi_{c}$ can help us to obtain a much clearer picture of the $J / \psi$ production. In addition to that, observables like the relative production of $\chi_{c 1}$ and $\chi_{c 2}$ serve as sensitive probes of the prompt charmonium production complementary to the studies of the $S$-wave states. ATLAS has measured differential cross-sections of prompt and non-prompt $\chi_{c 1}$ and $\chi_{c 2}$ production within the $J / \psi$ daughter rapidity region $\left|y^{J / \psi}\right|<0.65$ [11].

Fig. 7 shows the mass difference $\Delta_{m}=m\left(\mu^{+} \mu^{-} \gamma\right)-m\left(\mu^{+} \mu^{-}\right)$distribution for $\chi_{c}$ candidates. The peaks for the prompt and non-prompt production have been extracted using a simultaneous fit to the pseudo-proper time distribution. The differential cross-section of prompt $\chi_{c 1}$ production plotted as a function of $p_{\mathrm{T}}^{J / \psi}$ can be seen in Fig. 8 (left). The model predictions are also shown and one can conclude that NLO NRQCD describes the data very well. The same plot for the nonprompt production is in Fig. 8 (right). The FONLL model works here quite satisfactorily. Values of the cross-section ratios of prompt $\chi_{c 2}$ relative to prompt $\chi_{c 1}$ are displayed in Fig. 9. Here the NLO NRQCD predictions describe the data well up to $17 \mathrm{GeV}$. The LO CS model predicts consistently much lower values across the whole $p_{\mathrm{T}}$ range.

\subsection{Associated Production of Prompt $J / \psi+W^{ \pm}$}

The study of vector boson production in association with a $J / \psi$ represents an important tool to test QCD at the perturbative/non-perturbative boundary. Precise cross-sections measurements can shed more light on the contributions of colour-singlet and colour-octet states in heavy quarkonium production. Furthermore, the production is sensitive to multi-parton interactions in the collision. Recent studies have also shown the importance of these measurements in probing the Higgs boson charm couplings. Associated production of $J / \psi+W^{ \pm}$can also be an indication of a signature of a charged Higgs boson decay in some supersymmetry models. ATLAS has observed for the first time the production of $W^{ \pm}$in association with a prompt $J / \psi$ meson in the $W^{ \pm}\left(\rightarrow \mu^{ \pm} v_{\mu}\right)+J / \psi(\rightarrow$ $\mu^{+} \mu^{-}$) channel [15]. 


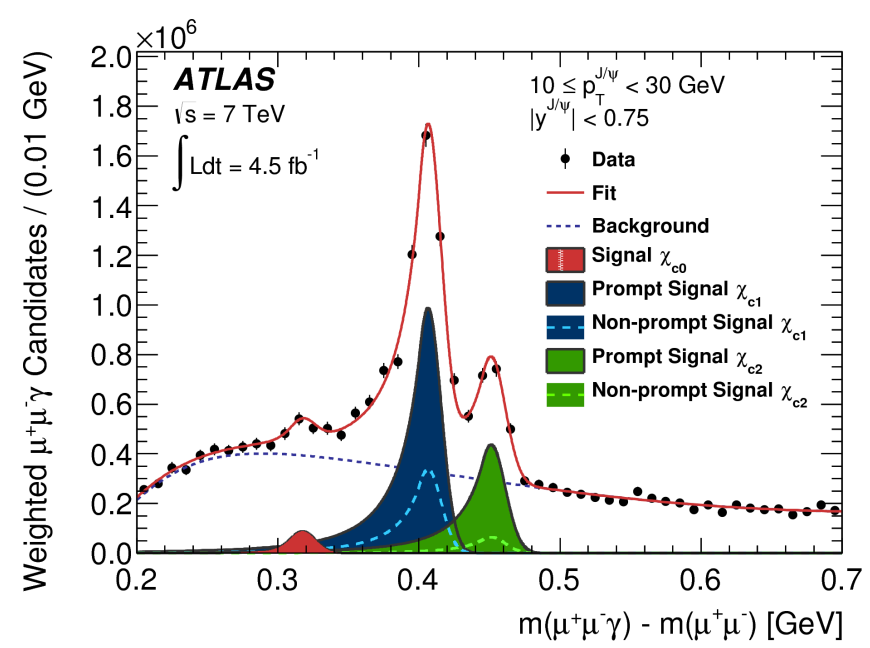

Figure 7: The mass difference $\Delta_{m}=m\left(\mu^{+} \mu^{-} \gamma\right)-m\left(\mu^{+} \mu^{-}\right)$distribution for $\chi_{c}$ candidates.
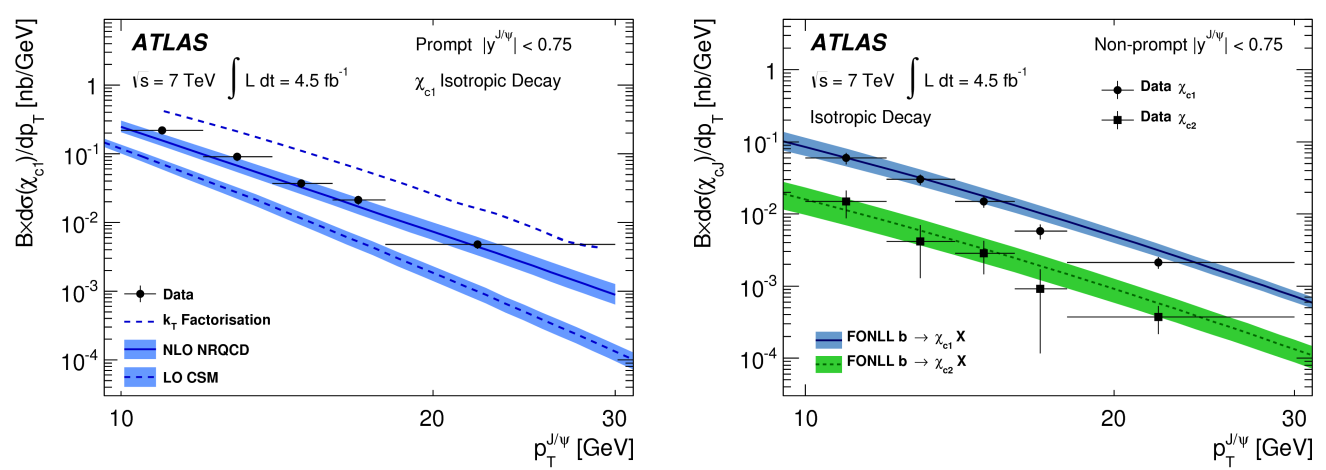

Figure 8: Differential cross-sections for prompt (left) and non-prompt (right) $\chi_{c 1}$ production. The factor $B$ denotes the product of branching fractions, $B=\mathcal{B}\left(\chi_{c J} \rightarrow J / \psi \gamma\right) \cdot \mathcal{B}\left(J / \psi \rightarrow \mu^{+} \mu^{-}\right)$.

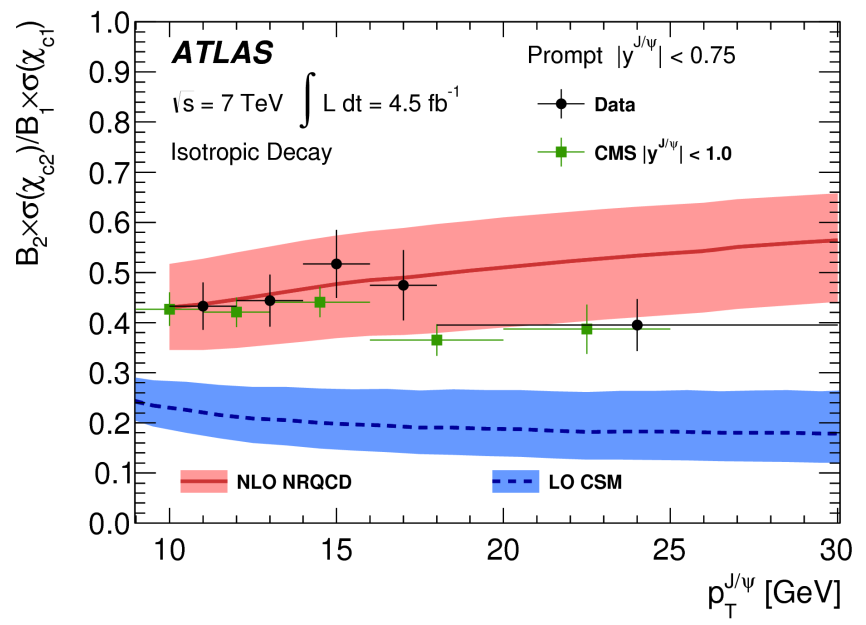

Figure 9: The production cross-section of prompt $\chi_{c 2}$ relative to prompt $\chi_{c 1}$. The factors $B_{1}$ and $B_{2}$ denote the branching fractions $B_{1}=\mathcal{B}\left(\chi_{c 1} \rightarrow J / \psi \gamma\right)$ and $B_{2}=\mathcal{B}\left(\chi_{c 2} \rightarrow J / \psi \gamma\right)$, respectively. 
The prompt component of the associated production cross-section relative to the inclusive $W^{ \pm}$ cross-section with different corrections applied is shown in Fig. 10 (left). The theory predictions are shown as well. An important aspect of this measurement is the estimate of the double parton scattering (DPS) contribution in the process. Its size can be seen in Fig. 10 (right), where the inclusive (SPS+DPS) cross-section is shown together with the DPS estimate. The data suggest that the single parton production (SPS) is the dominant production mode at low $J / \psi$ transverse momenta.
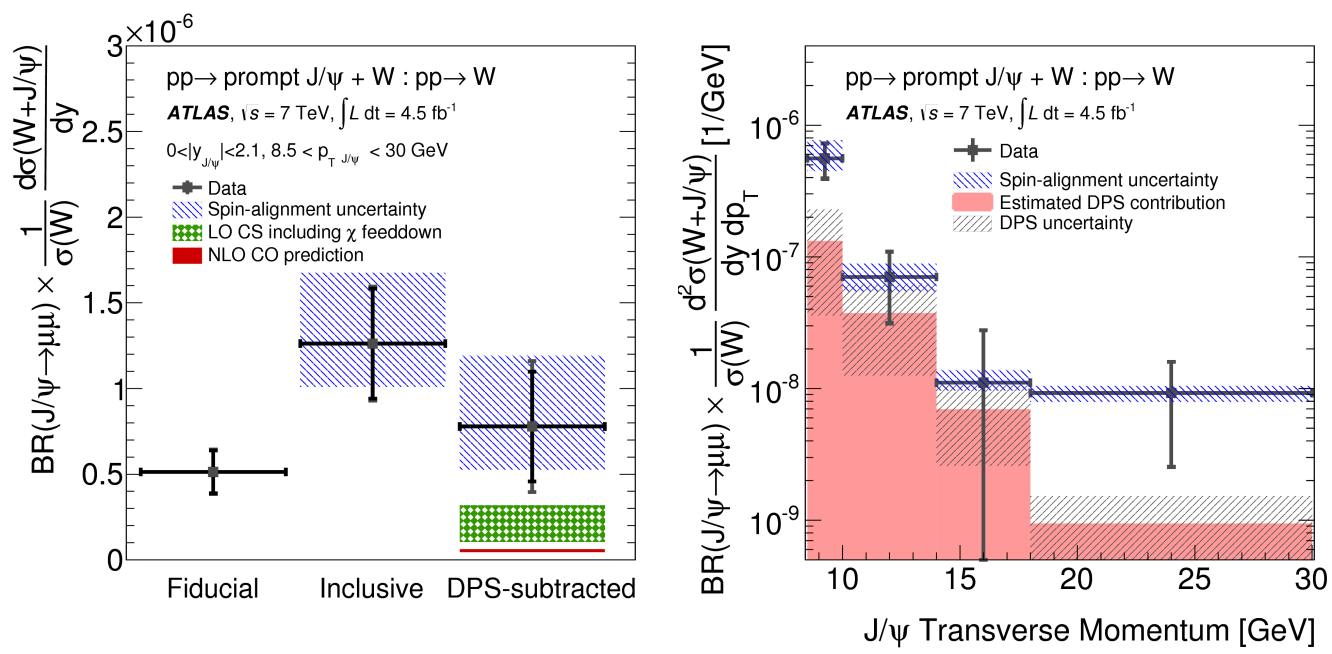

Figure 10: The prompt component of the associated production cross-section relative to the inclusive $W^{ \pm}$ cross-section (left). The inclusive (SPS+DPS) cross-section (right). For the explanation of the symbols see original paper [15].

\subsection{Associated Production of $J / \psi+Z^{0}$}

Similarly to the associated $W$ production, associated production of $J / \psi$ with a $Z^{0}$ boson is very promising area for the quarkonium production model tests. ATLAS has reported the first measurements for both prompt and non-prompt $J / \psi$ production [16]. The measurement is based on the $20.3 \mathrm{fb}^{-1}$ data collected at $\sqrt{s}=8 \mathrm{TeV}$. Also here, the contributions of SPS and DPS are separated. The normalised production cross-sections of this process can be seen in Fig. 11. The left plot shows results for the prompt production, together with DPS estimates and theoretical predictions. The results for the non-prompt production are shown in the right plot. The measurement has $5 \sigma$ significance for prompt production, and $9 \sigma$ significance for the non-prompt production.

\section{Conclusions}

A brief overview of the ATLAS flavour physics results has been presented. An up-to-date list of results and references to their descriptions can be found at the dedicated web page [17].

\section{References}

[1] ATLAS Collaboration, The ATLAS Experiment at the CERN Large Hadron Collider, JINST 3 S08003 (2008) 

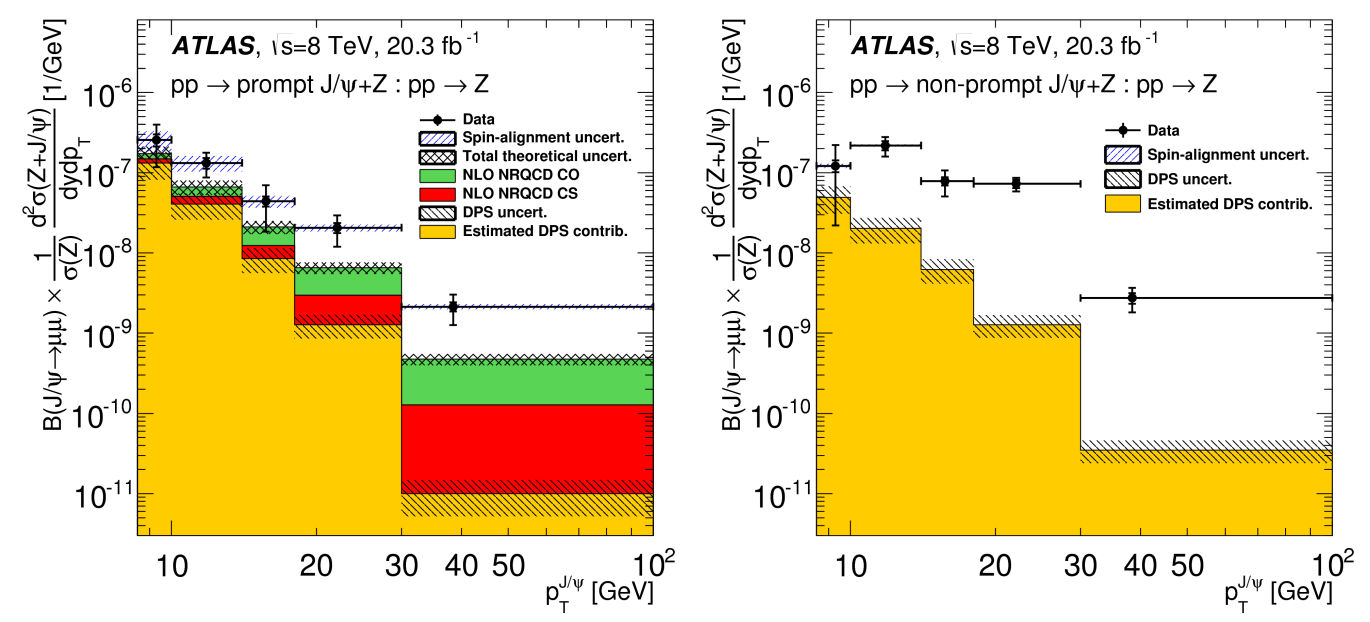

Figure 11: The $J / \psi+Z^{0}$ production cross-section as a function of the $p_{\mathrm{T}}$ of prompt (left) and non-prompt (right) $J / \psi$.

[2] CDF Collaboration, F. Abe, et al., Observation of the $B_{c}$ Meson in $p \bar{p}$ Collisions at $\sqrt{s}=1.8 \mathrm{TeV}$, Phys. Rev. Lett. 81 (1998) 2432

[3] ATLAS Collaboration, Observation of an Excited $B_{c}^{ \pm}$Meson State with the ATLAS Detector, Phys. Rev. Lett. 113, 212004

[4] HFAG, Y. Amhis et al., Averages of $b$-hadron, $c$-hadron, and $\tau$-lepton properties as of summer 2014, arXiv: 1412.7515

[5] ATLAS Collaboration, Flavor tagged time-dependent angular analysis of the $B_{s}^{0} \rightarrow J / \psi \phi$ decay and extraction of $\Delta \Gamma_{s}$ and the weak phase $\phi_{s}$ in ATLAS, Phys. Rev. D 90 (2014) 052007

[6] Belle Collaboration, S.-K. Choi, et al., Observation of a narrow charmoniumlike state in exclusive $B^{ \pm} \rightarrow K^{ \pm} \pi^{+} \pi^{-} J / \psi$ decays, Phys. Rev. Lett. 91 (2003) 262001

[7] BaBar Collaboration, B. Aubert, et al., Study of the $B^{-} \rightarrow J / \psi K^{-} \pi^{+} \pi^{-}$decay and measurement of the $B^{-} \rightarrow X(3872) K$ branching fraction, Phys. Rev. D 71 (2005) 071103

[8] CDF Collaboration, D. Acosta, et al., Observation of the Narrow State $X(3872) \rightarrow J / \psi \pi^{+} \pi^{-}$in $\bar{p} p$ Collisions at $\sqrt{s}=1.96 \mathrm{TeV}$, Phys. Rev. Lett. 93 (2004) 072001

[9] D0 Collaboration, V. Abazov, et al., Observation and Properties of the $X$ (3872) Decaying to $J / \psi \pi^{+} \pi^{-}$in $p \bar{p}$ Collisions at $\sqrt{s}=1.96 \mathrm{TeV}$, Phys. Rev. Lett. 93 (2004) 162002

[10] ATLAS Collaboration, Search for the $X_{b}$ and other hidden-beauty states in the $\pi^{+} \pi^{-} \Upsilon(1 S)$ channel at ATLAS, Phys. Lett. B740 (2015) 199-217

[11] ATLAS Collaboration, Measurement of $\chi_{c 1}$ and $\chi_{c 2}$ production with $\sqrt{s}=7 \mathrm{TeV} p p$ collisions at ATLAS, JHEP 07 (2014) 154

[12] ATLAS Collaboration, Measurement of the production cross-section of $\psi(2 S) \rightarrow J / \psi\left(\rightarrow \mu^{+} \mu^{-}\right)+\pi^{+} \pi^{-}$in $p p$ collisions at $\sqrt{s}=7 \mathrm{TeV}$ at ATLAS, JHEP 09 (2014) 079

[13] CMS Collaboration, S. Chatrchyan, et al., $J / \psi$ and $\psi(2 S)$ production in $p p$ collisions at $\sqrt{s}=7 \mathrm{TeV}$, JHEP 02 (2012) 011 
[14] LHCb Collaboration, R. Aaij, et al., Measurement of $\psi(2 S)$ meson production in $p p$ collisions at $\sqrt{s}=7 \mathrm{TeV}, \mathrm{EPJC}, 72(2012) 2100$

[15] ATLAS Collaboration, Measurement of the production cross section of prompt $J / \psi$ mesons in association with a $W^{ \pm}$boson in $p p$ collisions at $\sqrt{s}=7 \mathrm{TeV}$, JHEP 04 (2014) 172

[16] ATLAS Collaboration, Associated production of prompt and non-prompt $J / \psi$ mesons and $Z$ boson at $\sqrt{s}=8 \mathrm{TeV}$, arXiv:1412.6428, submitted to EPJC

[17] ATLAS Collaboration, ATLAS Experiment - Public Results, B-physics public results, https://twiki.cern.ch/twiki/bin/view/AtlasPublic/BPhysPublicResults 